\title{
Contents of Volume 49 / Inhalt von Jahrgang 49 Sommaire du volume 49 / Indice dell'anno 49
}

Lazare Benaroyo, Les recherches de Theodor Kocher sur l'étiologie de l'ostéomyélite et de la strumite aiguës (Theodor Kocher's research on the aetiology of acute osteomyelitis and strumitis)

René Bernoulli, Montaigne und Paracelsus (Montaigne and Paracelsus)

Christian Bryois, Theodor Kocher et César Roux (Theodor Kocher and César Roux)

Bernardino Fantini, La révolution pastorienne et les théories sur l'étiologie du goitre et du crétinisme (The bacteriological revolution and the theories on the aetiology of goiter and cretinism)

Huldrych M. Koelbing, Zum Columbus-Zentenarium 1492-1992 (Editorial) ( The Columbus Year, 1492-1992)

Jürgen Konert, S.A.Tissot und sein Einfluss auf den «DessauWörlitzer Kulturkreis» (Tissot's influence in Anhalt-Dessau)

Urs B. Leu, Konrad Gessner und die Neue Welt (Conrad Gessner and the New World)

Dorothea Liebermann-Meffert, Martin Alggöwer, Thomas RüEDI, Die Beziehungen Theodor Kochers zur «Société Internationale de Chirurgie» - Seine Rolle als erster Kongress-Präsident (Theodor Kocher and the International Society of Surgery-his role as the chairman of its first congress)

Christoph Mörgeli, Zürcher Lazarette in den Kriegen von 1798/99Ein Manuskript von Spitalarzt Johann Ludwig Meyer

Michel Porret, La «mort de la belle jeunesse» ou le suicide juvénile à Genève au XVIII ${ }^{\mathrm{e}}$ siècle (Juvenile suicide at Geneva in the 18th century)

Loris Premuda, Die Präsenz Theodor Kochers im Werk des Triestiner Chirurgen Gustavo Usiglio über die Schilddrüsentumoren (1894) (Theodor Kocher's presence in the book of Gustavo Usiglio of Trieste on thyroid tumours, 1894)

Plinio Prioreschi, Did the Hippocratic physician treat hopeless cases? 
Kurt Ritzmann, Theodor Kocher und Zürich (Theodor Kocher and Zurich)

Wilfried Schröder und Karl Heinrich Wiederkehr, Georg von Neumayer (1826-1909) und die internationale Entwicklung der Geophysik (Georg von Neumayer [1826-1909] and international geophysics)

Selwyn Taylor, Surgery for exophthalmic goitre in Australia, 1907

Ulrich Tröhler, Theodor Kocher: Chirurgie und Ethik (Theodor Kocher: surgery and ethics)

JÜrg ULRIGH, Ludwik Fleck (1896-1961), Bakteriologe und Wissenschaftstheoretiker: Die Wissenschaft wächst im Denkkollektiv (Ludwik Fleck [1896-1961], bacteriologist and science theoretician : science grows in the thinking collective)

Peter Wegmann, Felix Meyer und Caspar Wolf. Anfänge der malerischen Entdeckung der Alpen (Felix Meyer and Caspar Wolf: the beginnings of the discovery of the Alps by the painters)

Richard B. Welbourn, The emergence of endocrinology

Constant Wieser, Theodor Kocher und die Anfänge der Radiologie in der Schweiz (Theodor Kocher and the beginnings of radiology in Switzerland)

Obituaries / Nachrufe / Nécrologie / Negrologia

Daniel Bovet, 1907-1992 (Bickel)

227

Gunter Mann, 1924-1992 (Kümmel)

385

Friedrich Rintelen, 1906-1991 (Koelbing)

Short communications / Kleine Mitteilungen

Petites communications / Brevi comunicazioni

Alfred Werner, the first Swiss Nobel Chemistry Laureate 234

Auszeichnung für eine medizinhistorische Studie

Centro Tedesco di Studi Veneziani - Das Deutsche Studienzentrum in Venedig (Bergdolt)

Landarzt vor 300 Jahren: der luzernische Chirurg und Geburtshelfer Klaus Melchior Bründler († 1721) (Schürmann)

Prix et Médaille Marc-Auguste Pictet 1992

Redaktion des GESNERUS 


\section{UNIVERSITY NeWS}

B. Fantini professeur d'histoire de la médecine à Genève $\quad 389$

Université de Lausanne $\quad 389$

Congresses, Meetings, Societies $\quad 65,391$

Henry-E.-Sigerist-Preis 1992

Jahresversammlung der Schweizerischen Gesellschaft für Geschichte der Medizin und der Naturwissenschaften, Basel, 2./3. Oktober 1992

Zu Paracelsus' 500. Geburtstag 392

\section{Essay Reviews}

Angela Graf-Nold, Zur Pathographie Friedrich Nietzsches (Besprechung von Pia Daniela Volz, Nietzsche im Labyrinth seiner Krankheit)

Jean Lindenmann, Ein Versuch, die Geschichte der Virologie zu schreiben (Besprechung von Alfred Grafe, A history of experimental virology)

Book Reviews / Buchbesprechungen / Critiques de livres / Recensioni

Georg Agricola, Bermannus (Le Mineur). Un dialogue sur les mines (Trümpy)

Jürg Ammann, Karl Studer (Hrsg.), 150 Jahre Münsterlingen. Das Thurgauische Kantonsspital und die Psychiatrische Klinik 1840-1990 (Boschung)

Christian Andree (Hrsg.), Über Griechenland und Troja, alte und junge Gelehrte, Ehefrauen und Kinder: Briefe von Rudolf Virchow und Heinrich Schliemann aus den Jahren 1877-1885 (Isler-Kerényi)

Marie-Louise Bauchot, L'ichtyologie en France au début du XIX'e siècle: l'histoire naturelle des poissons de Cuvier et Valenciennes (Ziswiler)

Axel Bauer, Die Krankheitslehre auf dem Weg zur Naturwissenschaftichen Morphologie (Böni)

Peter Emil Becker, Sozialdarwinismus, Rassismus, Antisemitismus und Völkischer Gedanke: Wege ins Dritte Reich, Teil II (Lindenmann)

Bruno Belhoste, Augustin-Louis Cauchy, a biography (Burckhardt)

Isaac Benguigui, Trois physiciens genevois et l'Europe savante. Les De la Rive, 1800-1920 (Dreifuss)

Therese Bhattacharya-Stettler, Nox Mentis : Die Darstellung von Wahnsinn in der Kunst des 19. Jahrhunderts (Walser)

Johanna Bleker, Heinz-Peter Schmiedebach (Hrsg.), Medizin und Krieg : vom Dilemma der Heilberufe, 1865 bis 1985 (Haffter)

Wolfgang Böhm, Einführung in die Wissenschaftsgeschichte des Pflanzenbaus (Nösberger)

Brigitt Yvonne Bohner, Zur Ausbildung und Tätigkeit der Zürcher Hebammen im 
Almuth Bruder-Bezzel, Die Geschichte der Individualpsychologie (Heinrich) 262

Brunonianism in Britain and Europe, ed. by W.F. Bynum and Roy Porter (Koelbing) 109

Caelius Aurelianus, Celeres passiones. Tardae passiones - Akute Krankheiten, Chronische Krankheiten. Hrsg. von Gerhard Bendz, übers. von Ingeborg Pape (Mudry) 345

Catalogo de la Biblioteca Historica del Hospital San Juan de Dios de Granada (Engeler) 402

Centre Jean-Palerne: Mémoires VI, Bibliographie des textes médicaux latins. Antiquité et haut moyen âge (Mudry)

Centre Jean-Palerne: Mémoires VIII, Etudes de médecine romaine (Mudry)

Edward D. Churchill, Wanderjahr. The education of a surgeon (Weindling)

Anna Rita Corsini et Maria Paula Segoloni, Medicinae Plinii Concordantiae (Mudry)

Alessandro Dini, Vita e organismo: le origini della fisiologia sperimentale in Italia (Bonuzzi)

Thomas Drucker (ed.), Perspectives on the history of mathematical logic (Engeler)

Forschungsfreiheit. Ein ethisches und politisches Problem der modernen Wissenschaft. Hrsg. von Helmut Holzhey, Ursula Pia Jauch, Hans Würgler (Bickel)

Kurt Goldammer, Der göttliche Magier und die Magierin Natur (Daems)

Peter Grotzer (Hrsg.), Liebe und Hass (Scharfetter)

Ove Hagelin, «The byrth of mankynde otherwyse named the womans booke». Embryology, obstetrics, gynaecology through four centuries (Seger)

Susanne Hahn, Achim Thom (Hrsg.), «Ergebnisse und Perspektiven sozialhistorischer Forschung in der Medizingeschichte» (Bickel)

Daniel Heller, Eugen Bircher, Arzt, Militär und Politiker (Koelbing)

Klaus Hentschel, Interpretationen und Fehlinterpretationen der speziellen und der allgemeinen Relativitätstheorie durch Zeitgenossen Albert Einsteins (Kiefer)

David Hilbert, Natur und mathematisches Erkennen (Burckhardt)

Ilse Jahn, Grundzüge der Biologiegeschichte (Druey)

Gundolf Keil und Paul Schnitzer (Hrsg.), Das Lorscher Arzneibuch und die frühmittelalterliche Medizin (Seiler)

Andreas Kleinert, Anton Lampa, 1868-1938 (Balmer)

François Ledermann (Hrsg.), Pharmazie im Umbruch: die Schweizer Apotheker im 19. Jahrhundert ... mit einem Nachdruck von Carl Emil Ringk von Wildenberg «Medicinisch-pharmaceutische Statistik der Schweiz» (Daems)

Louis Lewin, Durch die USA und Canada im Jahre 1887 : ein Tagebuch (Ritzmann)

M. J.van Lieburg, Frau und Depression. Impressionen aus der Geschichte einer Wechselbeziehung (Walser) 
Pauline M. H. Mazumdar, Eugenics, human genetics and human fertility (Schinzel)

Medizinhistorisches Institut der Universität Bern, Kranksein, Pflegen, Heilen in Bern vom Mittelalter bis heute (Heller)

Adrian Merlo, Edgar Michaelis (1890-1967), Kritiker Freuds (Müller)

Patrizia Micoli, Maurizio Rotoli, I capelli nell'Antico Egitto. Patologia, terapia, cosmesi nei testi medici (Stettler)

Christoph Mörgeli, Das Medizinhistorische Museum der Universität Zürich (Bickel)

Nicolás Monardes, La Historia Medicinal de las cosas que se traen de nuestras Indias Occidentales (1565-1574) (Engeler)

George Mora (ed.), Witches, devils, and doctors in the Renaissance: Johann Weyer, De praestigiis daemonum (Nutton)

Bruce T.Moran, The alchemical world of the German Court. Occult philosophy and chemical medicine in the circle of Moritz of Hessen, 1572-1632 (Daems)

108

Christian Müller (Hrsg.), Die Gedanken werden handgreiflich (Ernst)

Gerhard H. Müller, Johann Adam Pollich (1741-1780), «Medicinae Doctor» und Pflanzengeograph der Pfalz (Boschung)

Andreas P. Naef, The story of thoracic surgery: milestones and pioneers (Geroulanos)

Frank Nager, Der heilkundige Dichter : Goethe und die Medizin (von Engelhardt)

Jorge Navarro, La imagen de Ultramar en la medicina Valenciana del siglo XIX (KellerSchnider)

Nobel Laureates in Physiology or Medicine. A biographical dictionary (Bickel)

Hans Nothdurfter (Hrsg.), St. Prokulus in Naturns. Ergrabene Geschichte. Von den Menschen des Frühmittelalters und der Pestzeit (Seiler)

413

Kathryn M. Olesko, Physics as a calling (Kiefer)

421

Paul-Emile Pilet, Naturalistes et biologistes à Lausanne (Dreifuss)

404

Plinio Prioreschi, A history of medicine. Vol. I : Primitive and Ancient medicine (Stettler)

Georg Rechenauer, Thukydides und die hippokratische Medizin. Naturwissenschaftliche Methodik als Modell für Geschichtsdeutung (Lorenz)

Johann Georg Reicheneder, Zum Konstitutionsprozess der Psychoanalyse (Müller) 86

Paul Ridder, Im Spiegel der Arznei. Sozialgeschichte der Medizin (Schramm) 264

Lucille B. Ritvo, Darwin's influence on Freud (Dieckhöfer)

Erhard Rosner, Die Heilkunst des Pien Lu. Arzt und Krankheit in bildhaften Ausdrücken der chinesischen Sprache (Schramm)

Nicolaas A. Rupke (ed.), Vivisection in historical perspective (Mudry)

Sebastian Schellong, Künstliche Beatmung. Strukturgeschichte eines ethischen Dilemmas (Gattiker)

André Schluchter, Das Gösgeramt im Ancien Régime: Bevölkerung, Wirtschaft und Gesellschaft einer solothurnischen Landvogtei im 17. und 18. Jahrhundert (Hubler)

Gert Schubring (Hrsg.), «Einsamkeit und Freiheit» neu besichtigt: Universitätsreformen und Disziplinenbildung in Preussen als Modell für Wissenschaftspolitik im Europa des 19. Jahrhunderts (Hagner)

Alfredo Serrai, Conrad Gesner. A cura di Maria Cochetti (Leu)

René Sigrist, Les origines de la Société de Physique et d'Histoire Naturelle (1790-1822). La science genevoise face au modèle français (Stettler)

Otto Sonntag (ed.), The Correspondence between Albrecht von Haller and Horace-Bénédict 
Soranos d'Ephèse, Maladies des femmes, tome 1, livre 1. Texte établi, trad. et commenté par Paul Burguière, Danielle Gourevitch, Yves Malinas (Mudry)

Soranus von Ephesus, Soranus' Gynecology. Transl. with an introd. by Owsei Temkin (Müller-Landgraf)

Piet van Spijk, Definition und Beschreibung der Gesundheit (Scharfetter)

Friedrich Steinle, Newtons Entwurf «Über die Gravitation ... : ein Stück Entwicklungsgeschichte seiner Mechanik (van der Waerden)

John Stillwell, Mathematics and its history (Neuenschwander)

Ulrich Stoll, Das «Lorscher Arzneibuch», Ein medizinisches Kompendium des 8. Jahrhunderts (Codex Bambergensis Medicinalis 1) (Seiler)

Christian Thomas (Hrsg.), "Auf der Suche nach dem ganzheitlichen Augenblick». Der Aspekt Ganzheit in den Wissenschaften (Bickel)

The uses of experiment. Studies in the natural sciences. Ed. by David Gooding, Trevor Pinch, Simon Schaffer (Hentschel)

Enrico Valsangiacomo (Hrsg.), Zum Wohle der Kranken: das Schweizerische Rote Kreuz und seine Rolle in der Krankenpflegeausbildung 1882-1976) (Stettler)

Walter Vandereycken, Ron Van Deth, Rolf Meermann, Hungerkünstler, Fastenwunder, Magersucht (Ritzmann)

Francesca Vannozzi, Inventario del Patrimonio dell'Ateneo senese - Gli strumenti scientifici (Boschung)

Gustav Wagner, Andrea Mauerberger, Krebsforschung in Deutschland (Martz)

Françoise Waridel, Le premier institut suisse pour enfants sourds-muets. Une page d'histoire yverdonnoise (Heller)

Hans-Joachim Waschkies, Anfünge der Arithmetik im Alten Orient und bei den Griechen (Neuenschwander)

Andreas Wasilewski, Heilkunst im Spiegel von Apothekenstandgefässen und ihren Signaturen (Ledermann)

Peter-Christian Wegner, Franz Joseph Gall 1758-1828: Studien zu Leben, Werk und Wirkung (Walser)

André Weil, Souvenirs d'apprentissage (Burckhardt)

Burghard Weiss, Zwischen Physikotheologie und Positivismus: Pierre Prevost (17511839) und die korpuskularkinetische Physik der Genfer Schule (Sigrist)

Constant Wieser, Hans Etter, Josef Wellauer, Radiologie in der Schweiz. Zu ihrem 75jährigen Bestehen hrsg. von der Schweiz. Gesellschaft für Radiologie und Nuklearmedizin (Balmer)

Alexander Witzel, Ein Lesebuch zur Unterhaltung \& zur Belehrung für Ärzte (Böni)

Book Notices

$111,271,424$

Addresses of reviewers

$112,272,426$

Volume 49: Contents / Inhalt / Sommaire / Indice 